\title{
Ursolic Acid Suppresses Hepatitis B Virus X Protein-mediated Autophagy and Chemotherapeutic Drug Resistance
}

\author{
CHING-DONG CHANG ${ }^{1 *}$, PING-YUAN LIN ${ }^{2 *}$, JUE-LIANG HSU ${ }^{3}$ and WEN-LING SHIH ${ }^{3}$ \\ Departments of ${ }^{1}$ Veterinary Medicine and ${ }^{3}$ Biological Science and Technology, \\ National Pingtung University of Science and Technology, Pingtung, Taiwan, R.O.C.; \\ ${ }^{2}$ Research and Development Department, Golden Dapu Biotech Corp., Chiayi, Taiwan, R.O.C.
}

\begin{abstract}
Hepatitis $B$ virus $X(H B x)$ protein is a multifunctional oncoprotein that affects diverse cell activities via regulation of various host cell signaling pathways. The current investigation demonstrated that ursolic acid (UA), a pentacyclic triterpenoid, protected hepatoma cells and reduced HBx-mediated autophagy through modulation of Ras homolog gene family member A (RhoA). Low-level ectopic $H B x$ expression in Huh7 cells induced more significant autophagosome formation than high-level HBx expression. $H B x$ activated beclin-1 promoter and enhanced the beclin-1 protein expression under low HBx expression. Transcription factor AP-1 played an essential function in HBx-mediated beclin-1 promoter activation. Inhibition of RhoA and its downstream effector Rho-associated coiled-coil-containing protein kinase 1 (ROCK1) alleviated HBx-mediated autophagy significantly. Transiently-expressed HBx elicited an increased RhoA-GTP level, as well as phospho-ROCKl transient accumulation. Utilization of transactivation-deficient $H B x$ demonstrated that the transactivation activity of $H B x$ is required for autophagy induction. Furthermore, UA suppressed HBx-mediated RhoA activation, beclin-1 promoter activation and subsequent autophagy induction, while, most importantly, reversed $H B x$-induced anti-cancer drug resistance.
\end{abstract}

Hepatitis B virus (HBV) and hepatitis C virus (HCV) are predominant risk factors for primary liver cancers, as almost $80 \%$ of cases of hepatocellular carcinoma (HCC) are correlated with underlying chronic $\mathrm{HBV}$ and $\mathrm{HCV}$ infection

*These Authors contributed equally to this work.

Correspondence to: Wen-Ling Shih, Department of Biological Science and Technology, National Pingtung University of Science and Technology, 1, Shuefu Rd., Neipu, Pingtung 91201, Taiwan. Tel: +886 87703202 (Ext. 5192), Fax: +886 87740550, e-mail: wlshih@mail.npust.edu.tw

Key Words: HBx, ursolic acid, autophagy, RhoA, ROCK1.
(1). Large population studies in Taiwan (2) and China (3) also showed that individuals with HCC have significantly higher seropositive results for hepatitis B surface antigen ( $\mathrm{HBsAg}$ ), indicating that chronic $\mathrm{HBV}$ infection is the key cause of HCC complications in these areas. HBV belongs to the Hepadnaviridae family and contains small, mostly doublestranded, circular DNA. HBV infection is initiated by the attachment and entry of virus particles into susceptible hepatocytes via cell surface receptors and endocytosis. Multiple host cell surface proteins are required for attachment and entry of HBV, although the mechanisms are not fully understood (4). In the treatment of patients with advanced HCC, systemic chemotherapy is the mainstay of therapy. The most active drugs for use in chemotherapy include doxorubicin, cisplatin and fluorouracil. Unfortunately, the positive response rate is only approximately $20 \%$ and these treatments have no significant effect on overall survival $(5,6)$.

The HBV-encoded X ( $\mathrm{HBx}$ ) protein modulates various cellular responses, including gene transcriptional control, signal transduction crosstalk, cell growth and programmed cell death, thus leading to genetic instability and carcinogenesis. Although still controversial, generally, in HBx-expressing hepatoma cells, cells confer anti-apoptosis and drug resistance in response to chemotherapeutic drugs (7). However, some reports have indicated that the expression level of $\mathrm{HBx}$ is correlated with the dual effects of $\mathrm{HBx}(8)$.

Autophagy, also called type II programmed cell death, is a cellular mechanism critical for homeostasis. More than 30 autophagy-related proteins (ATGs) have been identified from studies of yeast (9), with most being highly conserved from yeast to mammals. Beclin-1, the mammalian orthologue of yeast Atg6, plays a key role in autophagy. It also is a major component of the phosphatidylinositide 3-kinase (PI3K) class III lipid-kinase complex that induces autophagy and a substrate of Rho-associated coiled-coil-containing protein kinase 1 (ROCK1) that is phosphorylated during metabolic stress (10). Recent studies have shown that autophagy is a critical mechanism involved in liver disease. In some circumstances, autophagy suppresses tumorigenesis, while, 
in most cases, autophagy facilitates tumor progression (11). In several liver-derived cell lines, in a transgenic mouse model in addition to in the liver of HBV-infected patients, HBV induces autophagic flux and accumulates autophagic vacuoles. HBx has been shown to play a critical role in HBV-mediated autophagy. The possible mechanisms of the effects of HBx might be due to binding to type III PI3K and/or transactivation of the beclin-1 gene (12).

The search for naturally-existing compounds beneficial to health has continued in recent years, with a major focus on the constituents of plants being investigated for their anticancer activities (13). Ursolic acid (UA) and oleanolic acid (OA) are natural triterpenoid compounds that exist widely and can be enriched in herbs and plants. It is known that UA possesses multiple health-improving activities, including anti-oxidation, anti-inflammation, anti-cancer and hepatoprotective characteristics (14). Various combination chemotherapy regimens have also been studied. However, the precise mechanism underlying the hepatoprotective activity of UA still remains unclear.

Following a literature review, we investigated the relationship between autophagy and drug sensitivity response in $\mathrm{HBx}-$ expressing cells and evaluated the efficacy of UA treatment on HBx-expressing multidrug-resistant hepatoma cells; the cell signaling pathways involved were also elucidated.

\section{Materials and Methods}

Reagents and antibodies. Inhibitors, including Go66976 (PKC inhibitor), C3 exoenzyme (Rho inhibitor), Y27632 (ROCK inhibitor), LY294002 and wortmannin (PI3K inhibitors), PD98059 (ERK MAPK inhibitor), SP600125 (JNK inhibitor) and SB203580 (p38 MAPK inhibitor), were purchased from Calbiochem (San Diego, CA, USA). Autophagy inhibitor 3-methyladenine (3-MA) was also obtained from Calbiochem. Transfection was performed using Lipofectamine 2000 (Invitrogen, Carlsbad, CA, USA) according to the user's manual. Rabbit polyclonal antibody against HBx was provided by Dr. Shin-Lian Doong (National Taiwan University, Taipei, Taiwan) (15). Antibodies, including GAPDH, beclin-1, ROCK1 and phospho-ROCK1 were purchased from Cell Signaling Technology (Beverly, MA, USA). UA and OA were purchased from Sigma (St. Louis, MO, USA). Rho activator CN01 was obtained from Cytoskeleton, Inc. (Denver, CO, USA). Doxorubicin, etoposide and cisplatin were purchased from Sigma.

Expression plasmids. Dominant-negative Ras homolog gene family member A (RhoA) expression plasmid RhoA T-19N with myc-tag was kindly provided by Dr. Jin-Mei Lai (Fu-Jen Catholic University, Taipei, Taiwan). HBx expression plasmid with respiratory syncytial virus (RSV) promoter (RSV-HBx) and its empty control vector (RC$\mathrm{RSV}$ ), as well as inducible HBx point- and double-point mutation mutant expression plasmids, were kindly provided by Dr. Shin-Lian Doong (National Taiwan University, Taipei, Taiwan) $(15,16)$. Wildtype and three green fluorescent protein (GFP)-fused $\mathrm{HBx}$ mutants, pRT-HBxGFP, pRT-HBx ${ }^{7}$ GFP (amino acid 7; Cys changed to Ser), pRT-HBx ${ }^{61}$ GFP (amino acid 61; Cys changed to Leu) and pRT-HBx ${ }^{90-}$
${ }^{91}$ GFP (amino acid 90; Pro changed to Val and amino acid 91; Lys changed to Leu), were co-transfected with reverse tetracyclinecontrolled transactivator (rtTA) expression plasmid, pTRE-rtTA, and these recombinant plasmids generated doxycycline-inducible GFP fusion protein expression. pLC3-GFP (provided by Dr. Jen-Wei Lee, Tzu-Chi University, Hualien, Taiwan) expressed a fusion protein that allowed observation of autophagosome formation in cultured live cells.

Western blotting analysis. RhoA activation assay and ROCK activation assay. The procedures of Western blotting analysis and the RhoA activity assay were as described in the literature $(17,18)$.

Beclin-1 promoter controlled luciferase reporter plasmids and luciferase assay. A luciferase reporter controlled by a full-length promoter $(-644 /+197)$ and two deleted promoters $(-277 /+197)$ and ($528 /+197)$ was kindly provided Dr. Mujun Zhao (19). Analysis of the transcription factor binding site of the full-length (-644/+197) beclin1 promoter using an online system (20) revealed that there were three activator protein-1 (AP-1) binding sites in total and only one AP-1 binding site in a deleted beclin-1 promoter $(-277 /+197)$; however, no AP-1 binding site was present in the other deleted promoter $(-58 /+197)$. Site-directed mutagenesis on certain AP-1 binding sites was performed by Protech Technology Enterprise Co., Ltd. (Taipei, Taiwan). First, the consensus AP-1 binding sequence TGAC on a deleted promoter $(-277 /+197)$ was mutated to GTCG using sense primer 5'-AGATTACAGGCGGTCTCCACCGCGCCCGCCT-3' and antisense primer 5'-AGGCGGGCGCGGTGGAGACCGCCTGT AATCT-3'. This plasmid was named $\triangle \mathrm{AP}-1(-277 /+197)$. Two AP-1 binding sites on the full-length beclin-1 promoter $(-644 /+197)$ were mutated and 2 mutated plasmids were obtained. On the full-length beclin-1 promoter, the second AP-1 binding site located at -232/-237 was mutated using sense primer 5'-AGGTTCAAGCGATTCTCC GTAAGACGCCTCCCGAGTAGCTGG-3' and antisense primer 5'CCAGCTACTCGGGAGGCGTCTTACGGAGAATCGCTTGAACC T-3', and the plasmid was named $\triangle \mathrm{AP}-1-\mathrm{mt} 2(-644 /+197)$. The third AP-1 binding site of the full-length promoter located at -232/-237 was mutated using sense primer 5'-CTGAGATTACAGGCGGTCTAAC CCGCGCCCGCCTCCC-3' and antisense primer 5'-GGGAGGGG GCGCGGGTTAGACCGCCTGTAATCTCAG-3', with the resulting plasmid being named $\triangle \mathrm{AP}-1-\mathrm{mt} 3(-644 /+197)$. The three mutated nucleotides on the promoter were confirmed by direct DNA sequencing. Luciferase assay was performed according to the previous literature (18).

Inhibitor treatment of cells. Huh7 cells (kindly provided by Dr. Shin-Lian Doong, National Taiwan University, Taipei, Taiwan) were pre-treated with inhibitors at non-toxic concentrations for $2.5 \mathrm{~h}$, then plasmid DNA transfection was performed for a 24-h incubation period. Subsequently, $1.5 \mathrm{unit} / \mathrm{ml}$ Rho activator CN01 was added into the culture medium $1 \mathrm{~h}$ prior to harvesting of cells. C3 exoenzyme treatment was performed as described in our previous study (21). The complete C3 exoenzyme was delivered into the cells and plasmid DNA was then transfected, followed by incubation for a further $24 \mathrm{~h}$. Next, the activities of microtubule-associated protein 1 light chain 3 (LC3)-positive autophagosomes, beclin-1 promoter and ROCK were analyzed.

Half-maximal inhibitory concentration $\left(I_{50}\right)$ determination. Cell viability was determined by trypan blue exclusion assay. Cultured Huh7 cells were transfected with vector RC-RSV or $\mathrm{HBx}$ - 
expressing plasmid RSV-HBx for $24 \mathrm{~h}$ of incubation, then treated with anti-cancer drugs or drugs combined with UA, OA or 3methyladenine (3-MA) for an additional 24-h of incubation. Based on a series of dose-response data, the $\mathrm{IC}_{50}$ values were estimated using Excel software (Microsoft Corporation, Redmond, WA, USA).

Statistics. Three independent experiments were performed and each condition was tested in duplicate. Student's two-sample $t$-tests were conducted using the Analysis Tool Pack provided with Microsoft Excel. All $p$-values $<0.05$ were considered to indicate a significant difference.

\section{Results}

Kinetics of HBx expression and autophagy induction. To ensure that Huh7 cells were suitable for this study, plasmid pEGFP-C1, which encodes GFP, was used to test the transfection efficiency in the cells. The average transfection efficiency in the Huh7 cells was approximately $30-50 \%$, as observed under a fluorescent microscope at 48-72 h (data not shown). Next, the expression level of HBx in the Huh7 cells was examined by transfecting HBx plasmid with Rous sarcoma virus (RSV) promoter and it was found that the $\mathrm{HBx}$ protein expression gradually increased from 24-72 $\mathrm{h}$ post-transfection (Figure 1A). Using LC3-GFP plasmid, which contains LC3 autophagosome marker protein, autophagosome formation can be observed by monitoring the localization of the GFP signal, which represents cytoplasmic punctuation and indicates the autophagy phenomenon. In cells transfected with LC3-GFP and with or without HBx, the highest LC3-positive autophagosome formation was observed at $24 \mathrm{~h}$ post-transfection when the cells were cotransfected with $\mathrm{HBx}$. At this time point, the $\mathrm{HBx}$ protein expression level was still relatively low. With the increased $\mathrm{HBx}$ expression (Figure 1A), the percentage of autophagosome-positive cells decreased at 48 and $72 \mathrm{~h}$ posttransfection (Figure 1B). The diffuse fluorescence pattern revealed no autophagosome formation in cells without $\mathrm{HBx}$ transfection (Figure 1C, (a) and (c)), while GFP punctuation was seen in HBx-transfected cells, indicating autophagosome localization that represented cells undergoing autophagy (Figure 1C, (b)).

$H B x$ activates beclin-1 promoter via transcription factor $A P$ 1 and induces beclin-1 expression. A series of beclin-1 promoter constructs was used in this assay. Individual fulllength beclin-1 promoter $\mathrm{p}(-644 /+197)$ and two deleted beclin-1 promoters $\mathrm{p}(-277 /+197)$ and $\mathrm{p}(-58 /+197)$ were cotransfected into Huh7 cells with RSV-HBx plasmid. Figure $2 \mathrm{~A}$ indicates that $\mathrm{HBx}$ induced significant promoter activity of $\mathrm{p}(-277 /+197)$ and greater promoter activity of $\mathrm{p}(-$ $644 /+197)$; however, $\mathrm{HBx}$ was unable to activate $\mathrm{p}(-$ 58/+197). A luciferase assay using three AP-1 binding mutation reporters, including $\triangle \mathrm{AP}-1(-277 /+197), \Delta \mathrm{AP}-1-$ $\mathrm{mt} 2(-644 /+197)$ and $\Delta \mathrm{AP}-1-\mathrm{mt} 3(-644 /+197)$, demonstrated that $\mathrm{HBx}$ could not activate promoters without an AP-1 binding site (Figure 2A). No luciferase activity was observed in cells co-transfected with RC-RSV with all promoter constructions (data not shown). Further utilization of Western blotting analysis confirmed beclin-1 protein induction. The beclin-1 expression level gradually increased from 8-24 h, followed by a decrease from $48 \mathrm{~h}$ (Figure 2B).

RhoA plays a critical role in mediating $H B x$-induced autophagy and beclin-1 promoter activation. A panel of specific inhibitors was used to screen signal molecules possibly involved in the mediation of $\mathrm{HBx}$-induced autophagosome formation and beclin-1 promoter activation. The maximum non-cytotoxic concentrations of inhibitors used to treat Huh7 cells were determined by the MTT assay prior to performing the experiments (data not shown). Go6976 is a highly-effective inhibitor against protein kinase $\mathrm{C}$ (PKC) $\alpha$ and $\beta \mathrm{I}$ isoforms (22), the working concentration of which was $1 \mu \mathrm{M}$. C3 exoenzyme and RhoA T-19N were used to inhibit RhoA, though, due to a poor cell membrane permeability, C3 exoenzyme requires introduction into cells using Lipofectamine 2000 reagent $(18,21,23)$. The expression of dominant negative RhoA was examined using an antibody against myc-tag, as described in our previous study (18). Y27632 is a potent selective ROCK inhibitor, whereas LY294002 and wortmannin are two well-known inhibitors against phosphatidylinositol 3-kinase (PI3K). PD98059, SP600125 and SB203580 are mitogen-activated protein kinases (MAPK) pathway inhibitors that inhibit the activity of ERK, JNK and P38, respectively. The working concentration of wortmannin was $50 \mathrm{nM}$, while the working concentrations of Y27632, LY294002 and the three MAPKs inhibitors were $10 \mu \mathrm{M}$. As shown by the results presented in Figure 3, SB203580 had a moderate suppressive effect that reduced the percentage of autophagosome-positive cells and the full-length beclin-1 promoter activity $(p<0.05)$. Importantly, in the presence of C3 exoenzyme and dominant negative RhoA, and under the condition of inhibition of RhoA downstream effector ROCK, the suppression effects were even more evident $(p<0.01)$.

Up-regulation of RhoA and ROCKI activities upon $H B x$ expression. Having demonstrated the critical involvement of RhoA-dependent signaling, the status of RhoA and ROCK1 were then analyzed. Cells were transiently-transfected with empty vector (RC-RSV) or HBx-expression vector (RSVHBx) with RhoA treatment or ROCK1 inhibition. Pull-down assays revealed that low-level HBx expression (Figure 4A, top panel, lanes 6 and 7) induced an increase in the level of the GTP-bound form of RhoA in Huh7 cells 16 to 24 h posttransfection, whereas the level of total RhoA in cellular lysates was unchanged (Figure 4A, lower panel). The effect 
A

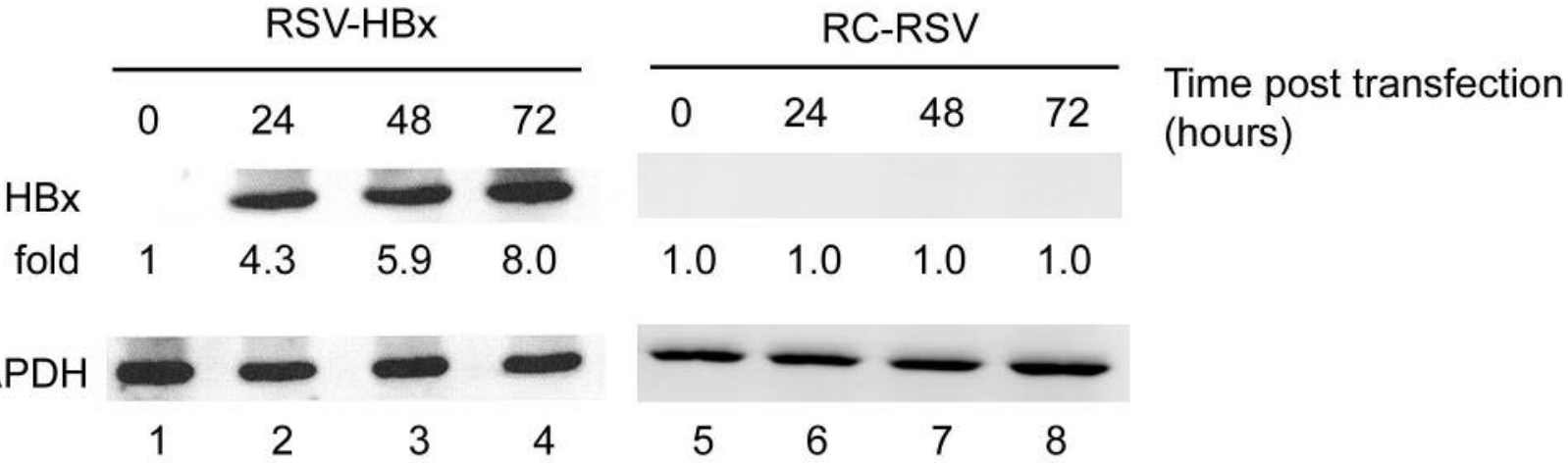

B

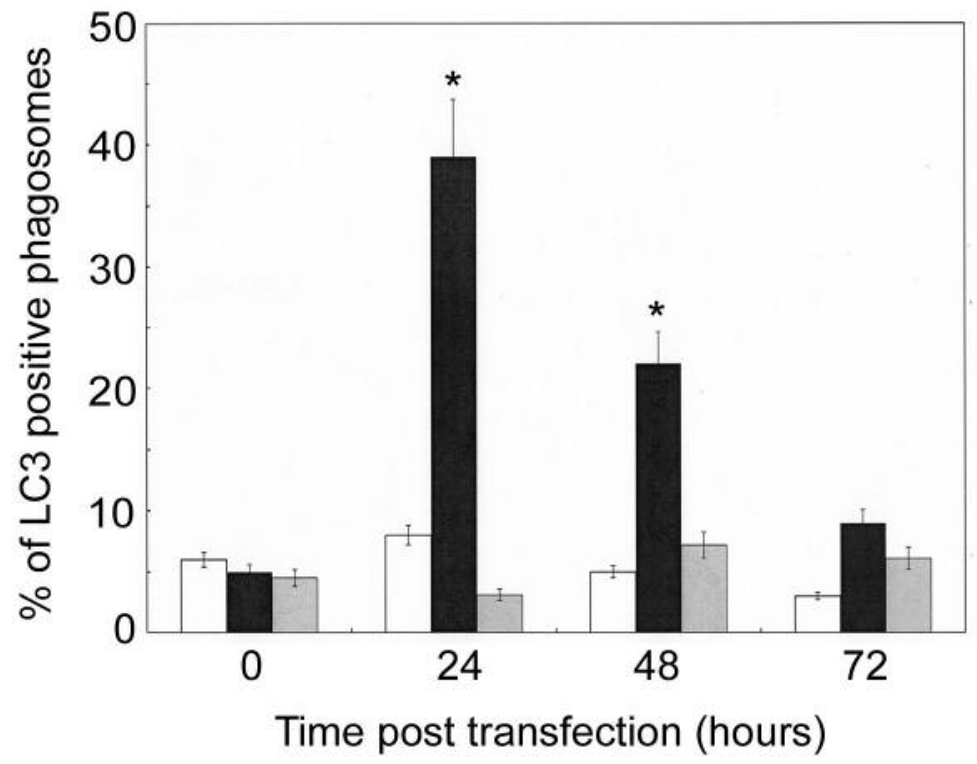

LC3-GFP

LC3-GFP+RSV-HBx

LC3-GFP+RC-RSV

C

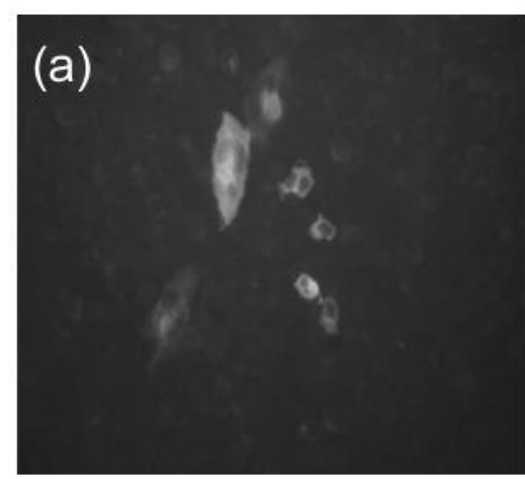

LC3-GFP

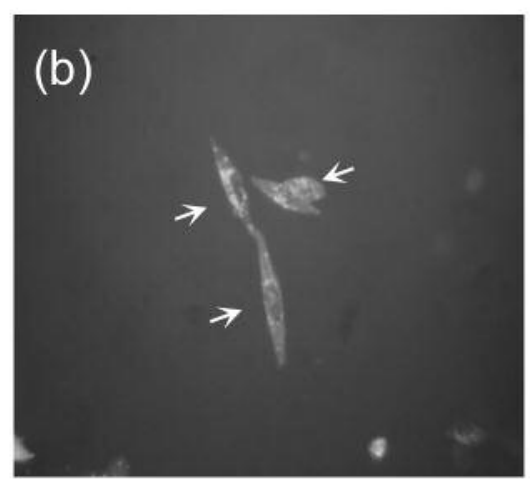

LC3-GFP+RSV-HBx

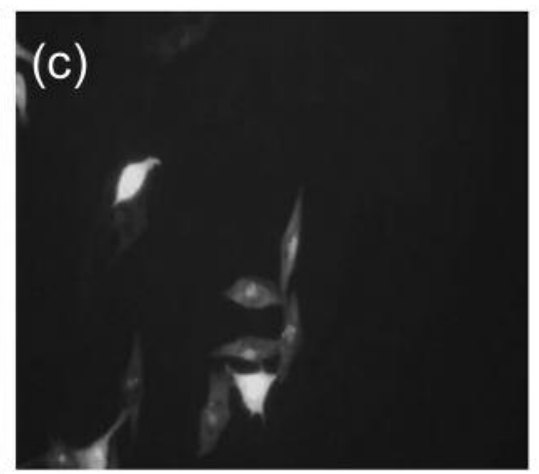

LC3-GFP+RC-RSV

Figure 1. Induction of autophagy by transient-expressed HBx. (A) Detection of transient HBx expression by western blotting analysis. Cultured $H u h 7$ cells were transfected with RC-RSV vector or HBx-expression plasmid RSV-HBx for different incubation durations. HBx was shown to be present in cells transfected with RSV-HBx as expected. GAPDH was included as an internal control. The relative HBx expression was normalized against " 0 " and is shown as the fold induction. (B) Time-course monitoring of LC3 dot-positive cells under different transfection conditions. *Significantly different compared to "O". (C) GFP fluorescence observation in Huh7 cells transfected with plasmid as indicated. White arrows show punctuation representing autophagosome formation. 


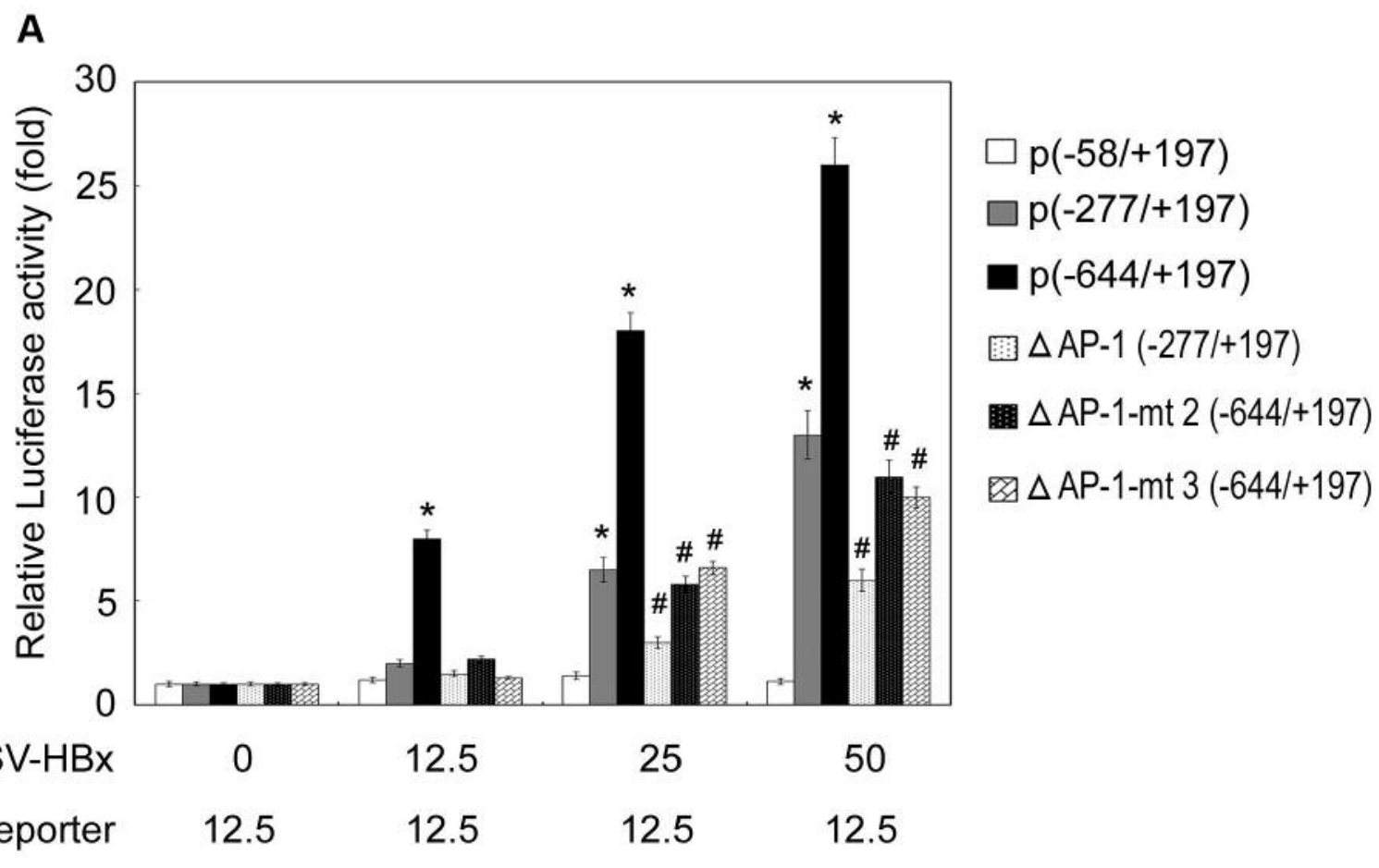

(ng)

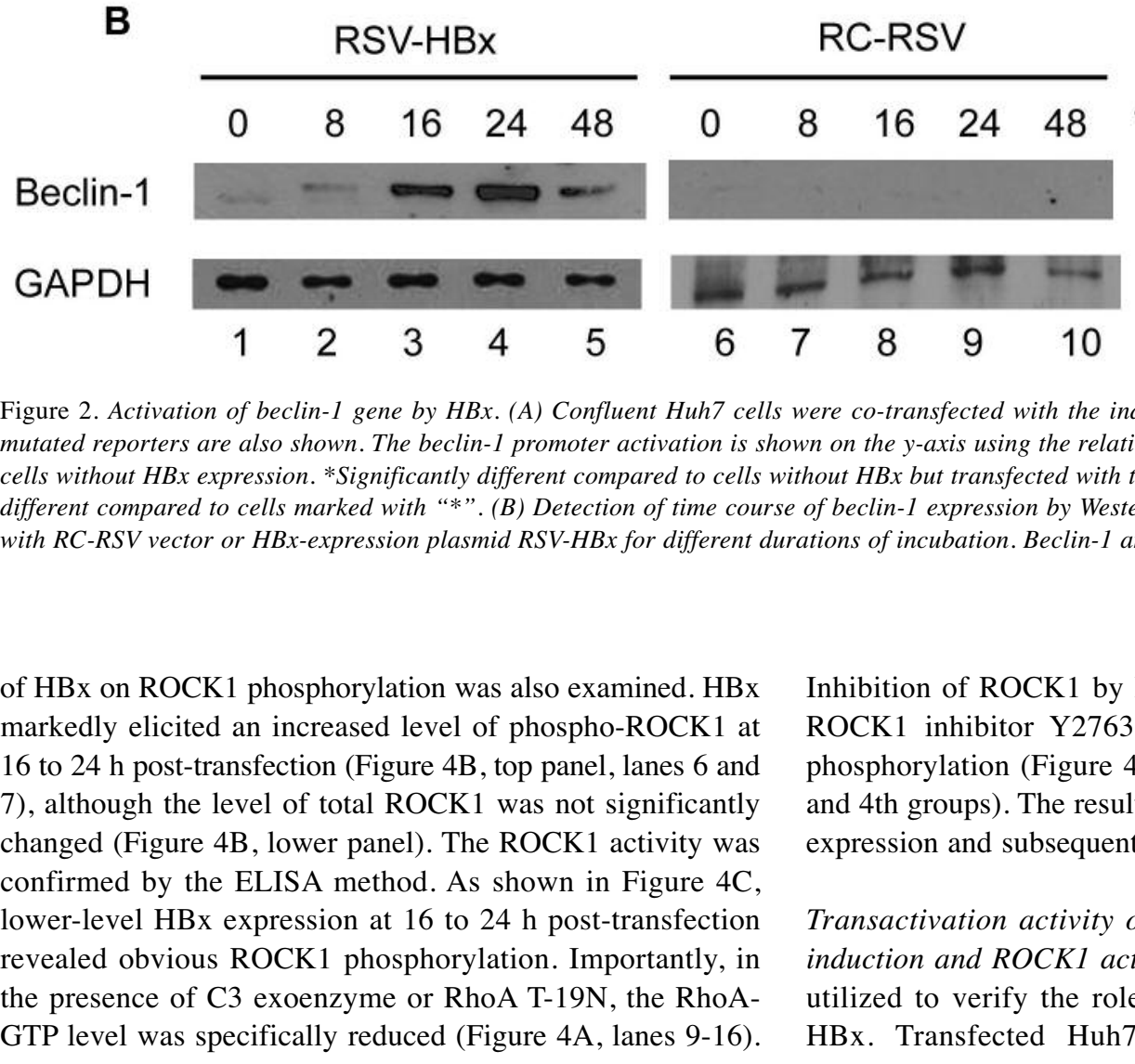

RC-RSV

Figure 2. Activation of beclin-1 gene by HBx. (A) Confluent Huh7 cells were co-transfected with the indicated plasmids. Various wild-type and mutated reporters are also shown. The beclin-1 promoter activation is shown on the y-axis using the relative luciferase activity normalized against cells without HBx expression. *Significantly different compared to cells without HBx but transfected with the same reporter plasmid. \#Significantly different compared to cells marked with “*”. (B) Detection of time course of beclin-1 expression by Western blotting. Huh7 cells were transfected with RC-RSV vector or HBx-expression plasmid RSV-HBx for different durations of incubation. Beclin-1 and GAPDH expression levels are shown.

of HBx on ROCK1 phosphorylation was also examined. HBx markedly elicited an increased level of phospho-ROCK1 at 16 to $24 \mathrm{~h}$ post-transfection (Figure 4B, top panel, lanes 6 and 7), although the level of total ROCK1 was not significantly changed (Figure 4B, lower panel). The ROCK1 activity was confirmed by the ELISA method. As shown in Figure 4C, lower-level HBx expression at 16 to $24 \mathrm{~h}$ post-transfection revealed obvious ROCK1 phosphorylation. Importantly, in the presence of C3 exoenzyme or RhoA T-19N, the RhoAGTP level was specifically reduced (Figure 4A, lanes 9-16).
Inhibition of ROCK1 by RhoA inhibitor C3 exoenzyme or ROCK1 inhibitor Y27632 resulted in decreased ROCK1 phosphorylation (Figure 4B, lanes 9-16 and Figure 4C, 3rd and 4th groups). The results showed that $\mathrm{HBx}$ induced RhoA expression and subsequent ROCK1 signaling transmission.

Transactivation activity of $H B x$ is required for autophagy induction and ROCK1 activation. Three HBx mutants were utilized to verify the role of the transactivation ability of HBx. Transfected Huh7 cells expressing doxycycline- 
A

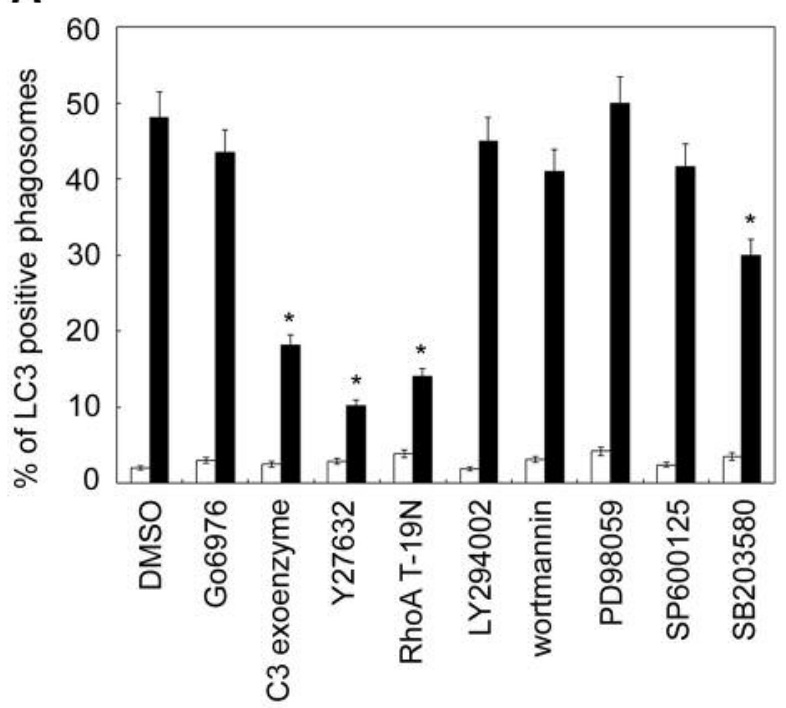

B

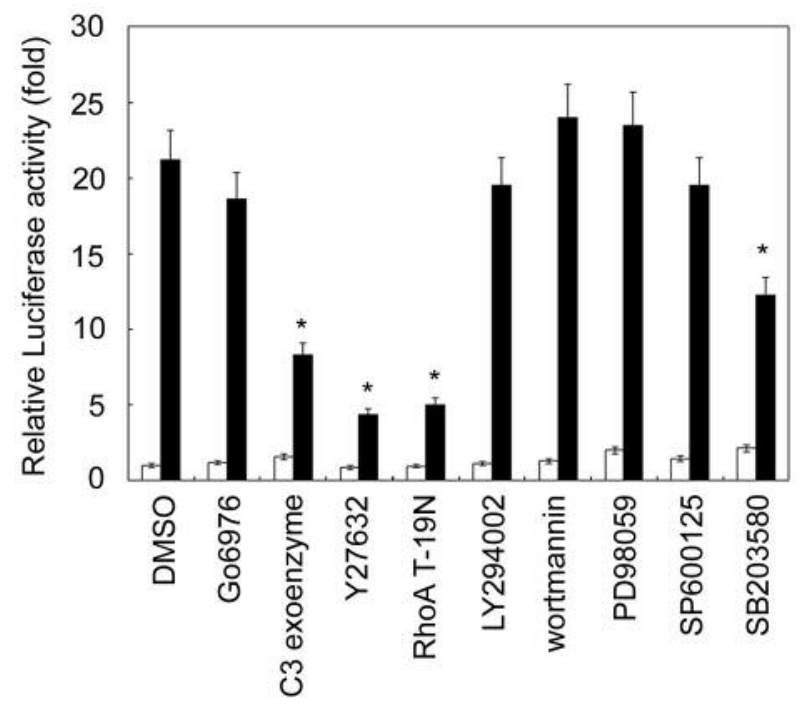

RC-RSV

RSV-HBx

Figure 3. Percentage of LC3-positive cells (A) and beclin-1 promoter activation fold $(B)$ in cells treated with various inhibitors with or without HBx expression. *Significantly different compared to cells without HBx expression.

inducible GFP and wild-type or mutated HBx-GFP fusion protein were analyzed by Western blotting, which confirmed the desired protein expression, as per the method employed in our previous study (15). GFP did not affect the HBxmediated transactivation on transcription factor AP-1 and nuclear factor-kappaB (NF-kB) (data not shown). $\mathrm{HBx}^{7} \mathrm{GFP}$
Table I. UA reverses the drug resistance induced by $H B x$.

\begin{tabular}{cccc}
\hline & Doxorubucin & Etoposide & Cisplatin \\
\hline RC-RSV & & & \\
DMSO & $1.22 \pm 0.09$ & $0.87 \pm 0.06$ & $0.31 \pm 0.03$ \\
3-MA & $1.10 \pm 0.08$ & $0.85 \pm 0.07$ & $0.33 \pm 0.03$ \\
RSV-HBx & & & \\
DMSO & $2.85 \pm 0.11^{*}$ & $2.01 \pm 0.12^{*}$ & $0.79 \pm 0.04^{*}$ \\
3-MA & $1.47 \pm 0.11^{\#}$ & $0.98 \pm 0.07^{\#}$ & $0.39 \pm 0.02^{\#}$ \\
RC-RSV & & & \\
UA & $1.26 \pm 0.12$ & $0.81 \pm 0.03$ & $0.29 \pm 0.02$ \\
OA & $1.32 \pm 0.12$ & $0.94 \pm 0.08$ & $0.34 \pm 0.04$ \\
RSV-HBx & & & \\
UA & $1.25 \pm 0.08^{\#}$ & $0.90 \pm 0.03^{\#}$ & $0.32 \pm 0.01^{\#}$ \\
OA & $2.92 \pm 0.17^{*}$ & $0.95 \pm 0.10^{*}$ & $0.81 \pm 0.07^{*}$ \\
\hline
\end{tabular}

Statistical significance of $\mathrm{IC}_{50}(\mu \mathrm{g} / \mathrm{ml})$ is indicated by “*” and “\#”. “*” represents data comparison with cells transfected with vector control RC-RSV and treated with DMSO. "Comparison with cells transfected with HBx-expressing plasmid RSV-HBx (groups 2 and 4).

possessed a transactivation ability, while $\mathrm{HBx}^{6 \mathrm{l}} \mathrm{GFP}$ and $\mathrm{HBx}^{90-91}$ GFP lost the transactivation function of HBx (24). The percentage of LC3 punctate cells, the full-length beclin1 promoter activity and ROCK 1 activity assays revealed that the transactivation function of $\mathrm{HBx}$ was closely correlated with the HBx-induced autophagy process (Figure 5A). Our results also demonstrated that $\mathrm{HBx}^{90-91}$ contributed the major transactivation function of $\mathrm{HBx}$.

UA inhibits RhoA and subsequent autophagy induction by $H B x$. UA and OA are pentacyclic triterpenoid acids that are widely considered to have a hepatoprotective function (25). Our previous study demonstrated that UA, but not OA, suppressed the $\mathrm{HBx}$-mediated tumorigenic effects (17). We then examined the possible effects of UA and OA on HBx-mediated autophagy induction. The results indicated that only UA suppressed HBxmediated autophagosome accumulation (Figure 5B, upper), beclin-1 promoter activation (Figure $5 \mathrm{~B}$, middle) and ROCK activity (Figure 5B, lower). Furthermore, in the presence of Rho activator $\mathrm{CN} 01, \mathrm{HBx}$-elicited autophagy-related effects were significantly increased (Figure 5B).

Reversion of HBx-mediated DNA-damage drug resistance by $U A$. Previous studies found that $\mathrm{HBx}$ endowed multiple chemotherapeutic drugs resistance through modulation of various cellular factors, for example, p53 tumor suppressor and NF-kB $(7,26,27)$. To further understand the therapeutic potential of UA, the drug sensitivity $\mathrm{IC}_{50}$ was evaluated in Huh7 cells treated with three anti-cancer DNA-damage agents in clinical use. First of all, the original drug sensitivity response in Huh7 cells was determined. Cells were transfected with either RC-RSV or RSV-HBx, then treated with the indicated 
A

C3 exoenzyme RhoA T-19N

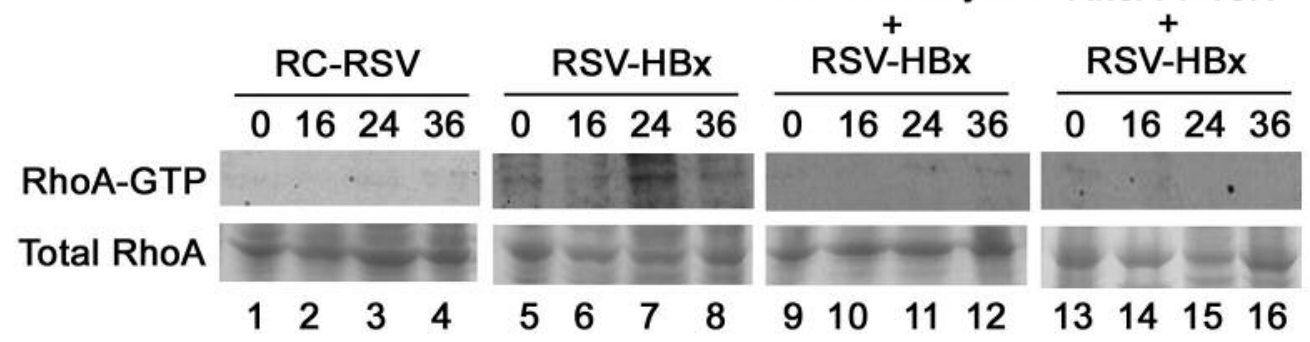

B

Y27632 C3 exoenzyme

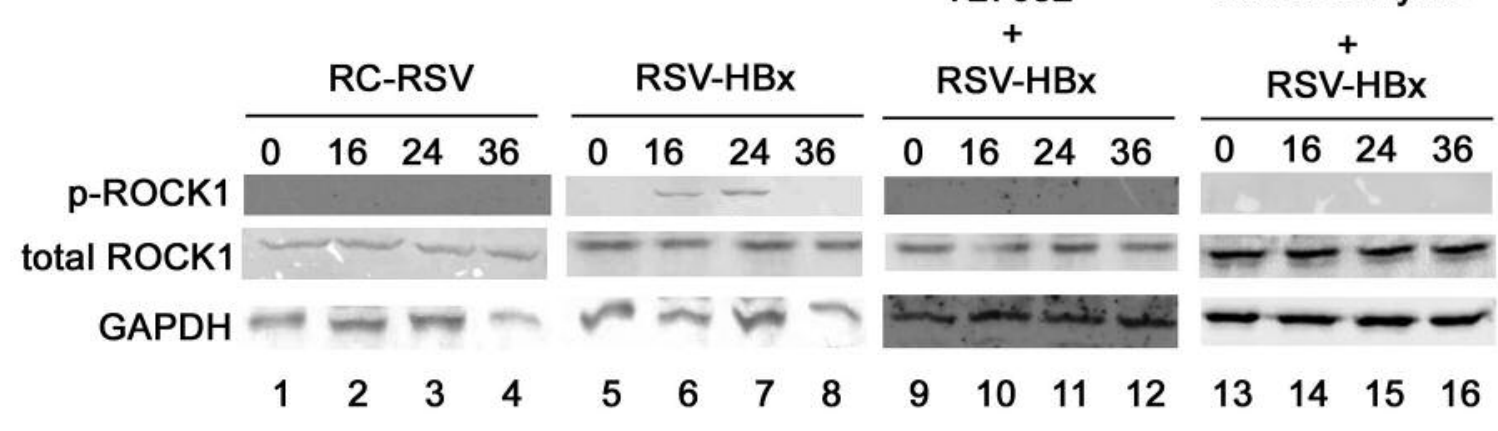

C

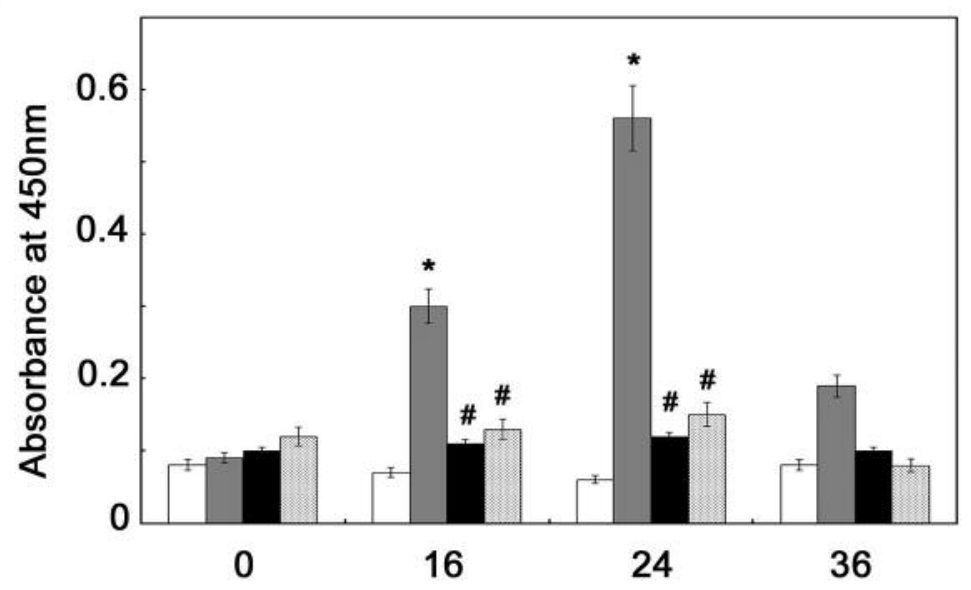

RC-RSV

RSV-HBx

RSV-HBX+Y27632

RSV-HBx+C3 exoenzyme

Time post transfection

(hours)

Figure 4. HBx activates RhoA and ROCK1 specifically. (A) Activation of RhoA by HBx. The level of GTP-bound RhoA was determined by pulldown assay; the level of total RhoA was not altered and is not shown. (B) The phosphorylation and total expression levels of ROCK-1 were monitored by Western blotting analysis. (C) ROCK1 activity was determined by ELISA. *HBx activation of ROCK1 compared to cells transfected with RCRSV. "Y27632 and C3 exoenzyme reversed the HBx-mediated ROCK1 activation effect.

compound. As shown in Table I, in the cells transfected with empty vector, the $\mathrm{IC}_{50}$ values of doxorubicin, etoposide and cisplatin were $1.22 \pm 0.09,0.87 \pm 0.06$ and $0.31 \pm 0.03 \mu \mathrm{g} / \mathrm{ml}$, respectively; in cells transfected with $\mathrm{HBx}$, the $\mathrm{IC}_{50}$ values of the three drugs were elevated by 2.3 - to 2.5 -fold.
To investigate the relationship between autophagy and drug response in our system, the well-known autophagy inhibitor 3-MA, which inhibits autophagy by blocking autophagosome formation via the suppression of type III PI-3K, was used. Blocking of autophagy by 3-MA in HBx-expressing cells also 
A
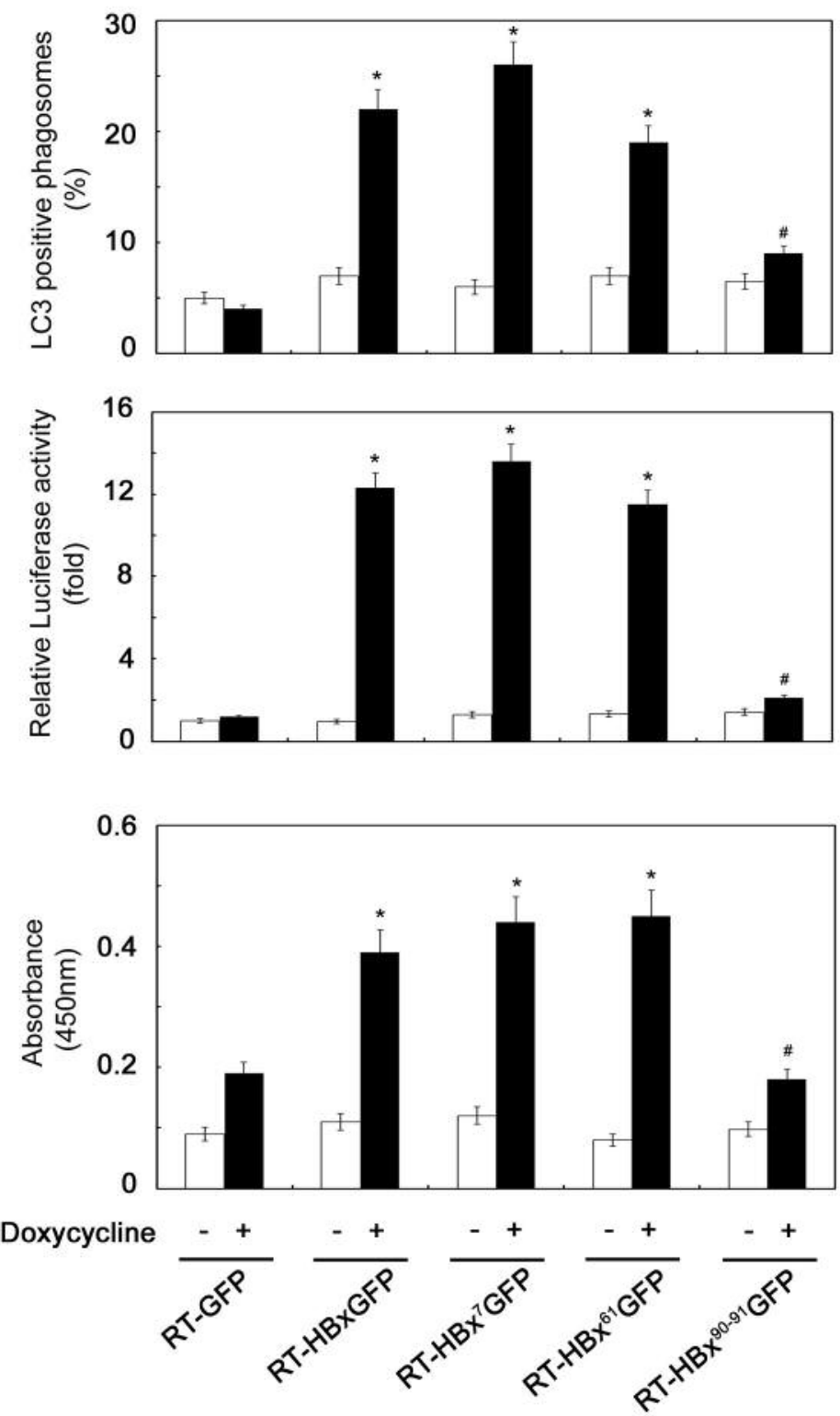

B
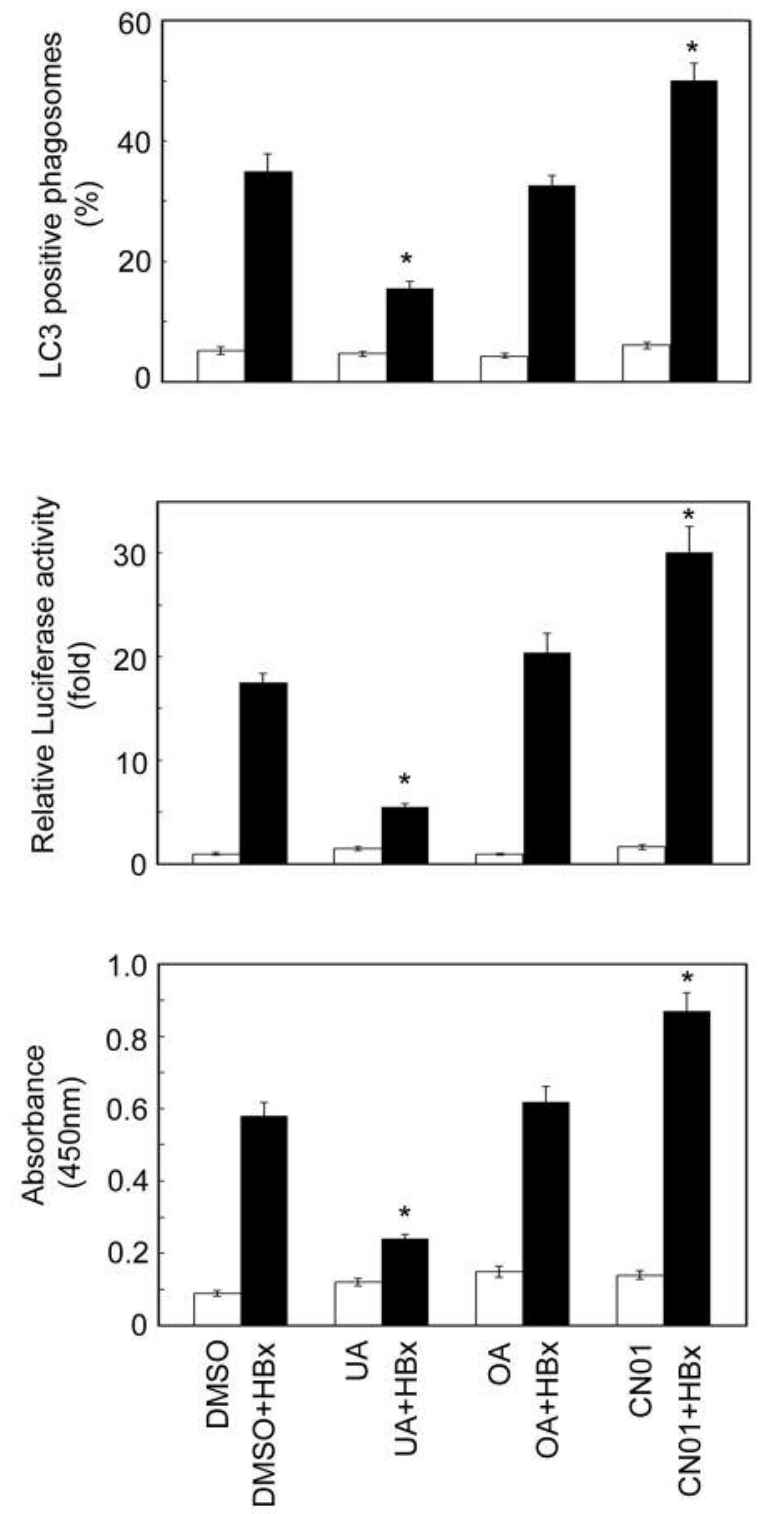

Figure 5. The transcriptional activation ability of HBx was indispensable for the HBx-mediated autophagy and ROCK activation. (A) The transactivation function of HBx was required for the induction of autophagy. The percentage of LC3-positive cells (upper), the beclin-1 promoter activity (middle) and the ROCK activity (lower) were measured in cells transfected with various HBx expression plasmids and treated with or without doxycycline. *Significantly different compared to cells with no doxycycline treatment. \#Comparison with “*”. (B) UA abolished the HBx-mediated autophagosome formation (upper), beclin-1 promoter activation (middle) and ROCK activation (lower), as shown by the significant differences marked "*” in group 2. RhoA activator CNO1 significantly increased the HBx-mediated effects, marked “*” in group 4.

significantly reduced the $\mathrm{IC}_{50}$ response against anti-cancer drugs (Table I, 3rd and 4th rows). Further experiments demonstrated that UA, but not OA, reversed the $\mathrm{HBx}-$ mediated drug resistance phenotype efficiently (comparing the 3rd and 7th rows, Table I) and, critically, the drug resistance reversion ability of UA was more efficient than that of 3-MA.

\section{Discussion}

The results of the present study indicated that expression of HBx protein increased punctate fluorescent signals in Huh7 cells co-transfected with GFP-tagged LC3, demonstrating that $\mathrm{HBx}$ is associated with autophagy. HBx expression 
activated beclin-1 promoter and enhanced the expressions of beclin-1, RhoA and ROCK1 proteins. Inhibition of RhoA and ROCK1 reduced HBx-induced autophagy.

Autophagy is an important dynamic process that maintains homeostasis of proteins and other macromolecules in cells. Damaged organelles and misfolded proteins are engulfed in autophagosomes that are double-membrane vesicles. During maturation to autolysosomes, the contents are degraded by lysosomal enzymes. The role of autophagy in cancer may be multifunctional. In the early stages of tumor development, it acts as a suppressor mechanism (28) promoting the recycling of damaged organelles and misfolded proteins, preventing oxidative stress and maintaining genome stability (29). However, certain types of tumors may require high levels of basal autophagy to maintain their tumorigenesis (30). Many studies have demonstrated that tumor cells may rely on autophagy for survival (31). The results of those studies indicated that autophagy may play roles in both anti-cancer and pro-cancer mechanisms.

Since most patients with HCC are being diagnosed with the disease at an advanced stage, they are not suitable candidates for liver transplantation. Surgical resection and ablation are only suitable for a small number of patients with single nodules or an early-stage tumor (32). As more than half of HCC cases develop due to chronic HBV infection, HBV infection-related liver diseases remain a global health problem. HBx protein is a multifunctional molecule that can stimulate $\mathrm{HBV}$ replication and is, therefore, considered to be important in the development of HBV-associated HCC (33). However, the role of HBx in the autophagy process has been poorly characterized to date.

LC3 is a protein that particularly associates with autophagosomes and, therefore, the autophagy level can be assessed by counting LC3-positive vesicles (34). The results of this study showed that the level of LC3-positive autophagosome synthesis was significantly increased in Huh7 cells at 24 and $48 \mathrm{~h}$ after $\mathrm{HBx}$ transfection and beclin-1 promoter activity and protein expression were also induced by HBx. We also found that the profiles of the percentage of LC3positive phagosomes under different treatments were of the same patterns as those of the beclin-1 promoter activity and/or ROCK activity (Figures 3, 5 and 6), indicating that beclin-1 and ROCK play key roles in autophagy. The importance of beclin-1 and ROCK was further supported by experiments using different inhibitors and dominant-negative RhoA (T-19N) mutant (Figure 3).

Autophagy and apoptosis are both regulated by common transcription factors and signaling pathways, such as tumor protein p53, PI3K/Akt, c-Jun N-terminal kinase (JNK) or NF$\mathrm{kB}$ (35). However, we found that inhibitors of PI3K and JNK did not affect $\mathrm{HBx}$-induced autophagy (Figure 3). In addition, inhibitors against PKC $\alpha$ and $\beta$ I isoforms (Go6976) (22) and MAPK (Erk) kinases (PD98059) also had no effect on autophagy. In our study, the beclin-1 expression level was correlated with the HBx expression kinetics, also being closely correlated with the kinetics of $\mathrm{HBx}$-induced autophagosome formation. Many studies have confirmed that beclin-1 regulates autophagy and that its expression level is correlated with the autophagy process $(36,37)$. Overexpression of beclin1 coincidentally increases the autophagosome level. As beclin-1 is a key component of autophagy and a substrate of ROCK1 (10), our results showed that inhibition of ROCK1 or upstream RhoA decreased the $\mathrm{HBx}$-induced autophagy. ROCK1 is known to mediate autophagy by binding beclin-1 and causing beclin-1 phosphorylation, which then disrupts beclin-1 and Bcl-2 interaction and triggers autophagy (10). Our results indicated that inhibition of RhoA and its downstream effector ROCK1 alleviated HBx-mediated autophagy significantly. Transiently-expressed HBx elicited an increased RhoA-GTP level, as well as phospho-ROCK1 transient accumulation. Utilization of transactivation-deficient $\mathrm{HBx}$ demonstrated that the transactivation activity of $\mathrm{HBx}$ is required for autophagy induction.

Studies have shown that p38 MAPK regulates the autophagy process in starvation-induced autophagy and myotube atrophy in muscle wastage by regulating certain ATG-related protein expressions (38). In our study, SB203580 treatment also resulted in a significant decrease in autophagy, suggesting that p38 MAPK is also involved in HBx-induced autophagy. However, p38 MAPK inhibitor had only a moderate suppression effect $(\sim 20 \%)$, which was weaker than the effects of Rho or ROCK inhibition ( 30 $40 \%$ ) in terms of the reduction of HBx-induced autophagy.

$\mathrm{Ke}$ and Chen demonstrated that HCV infection induces an unfolded protein response and the autophagy pathway, which are required to promote efficient viral replication (39). Shrivastava and colleagues found that knockdown of beclin1 and ATG7 reduced HCV RNA replication in immortalized human hepatocytes (40). These studies demonstrated a new mechanism employed by $\mathrm{HCV}$ to infect host cells. Although $\mathrm{HBV}$ and HCV share common clinical features, they are very different in terms of their molecular biology. HCV is a positive-sense single-stranded RNA virus, while $\mathrm{HBV}$ is a DNA virus that requires reverse transcription to replicate the DNA genomes. HBx protein is a candidate target for HVB treatment, as it is a transcriptional activator involved in the regulation of gene expression. Using gene sequence analysis (20), we identified several putative AP-1 binding sites on the beclin-1 promoter. In a luciferase assay, deletion or mutation of AP-1 sites demonstrated that AP-1 is a critical transcription factor for beclin-1 activation and plays an essential role in HBx-mediated beclin-1 promoter activation.

Several previous studies found that $\mathrm{HBx}$ induced multiple chemotherapeutic drugs resistance through regulating various signaling factors, such as p53 and NF-kB $(7,26,27)$. In our present study, the drug resistance activity induced by $\mathrm{HBx}$ 
was consistent with the results of several previous studies $(7$, $26,27)$; we identified that signaling pathways involving RhoA, ROCK1 and p38 MAPK are associated with HBxinduced autophagy. In addition, UA, a pentacyclic triterpenoid acid known to possess a hepatoprotective function, demonstrated a suppression effect on $\mathrm{HBx}$. Previously, we found that $\mathrm{HBx}$-mediated tumorigenic effects, including transcriptional activation, matrix metalloproteinase3 (MMP-3) secretion and anti-transforming growth factor- $\beta$ (TGF- $\beta$ ), induced apoptosis in hepatoma cells (17).

In light of the results of this and previous studies, we concluded that $\mathrm{HBx}$ activated RhoA and further transmitted the signal to RhoA downstream effector ROCK1. RhoA inhibition resulted in suppression of RhoA, as well as ROCK1. UA exhibited a protective effect in hepatoma cells by reducing the $\mathrm{HBX}$-induced autophagy and reversing the HBx-induced anti-cancer drug resistance. Our results suggested that UA has therapeutic potential for hepatoma treatment, especially in the circumstance of HBV infectioninduced drug resistance.

\section{References}

1 El-Serag HB: Epidemiology of viral hepatitis and hepatocellular carcinoma. Gastroenterology 142: 1264-1273 e1261, 2012.

2 Beasley RP, Hwang LY, Lin CC and Chien CS: Hepatocellular carcinoma and hepatitis B virus. A prospective study of 22707 men in Taiwan. Lancet 2: 1129-1133, 1981.

3 Evans AA, Chen G, Ross EA, Shen FM, Lin WY and London WT: Eight-year follow-up of the 90,000-person Haimen City cohort: I. Hepatocellular carcinoma mortality, risk factors, and gender differences. Cancer epidemiology, biomarkers \& prevention : A publication of the American Association for Cancer Research, cosponsored by the American Society of Preventive Oncology 11: 369-376, 2002.

4 Watashi K, Urban S, Li W and Wakita T: NTCP and beyond: Opening the door to unveil hepatitis B virus entry. Int J Mol Sci 15: 2892-2905, 2014.

5 Piguet AC, Saar B, Hlushchuk R, St-Pierre MV, McSheehy PM, Radojevic V, Afthinos M, Terracciano L, Djonov V and Dufour JF: Everolimus augments the effects of sorafenib in a syngeneic orthotopic model of hepatocellular carcinoma. Mol Cancer Ther 10: 1007-1017, 2011.

6 Tomuleasa C, Soritau O, Fischer-Fodor E, Pop T, Susman S, Mosteanu O, Petrushev B, Aldea M, Acalovschi M, Irimie A and Kacso G: Arsenic trioxide plus cisplatin/interferon alpha2b/doxorubicin/capecitabine combination chemotherapy for unresectable hepatocellular carcinoma. Hematol Oncol Stem Cell Ther 4: 60-66, 2011.

7 Liu Y, Lou G, Wu W, Shi Y, Zheng M and Chen Z: Interferonalpha sensitizes $\mathrm{HBx}$-expressing hepatocarcinoma cells to chemotherapeutic drugs through inhibition of HBx-mediated NFkappaB activation. Virol J 10: 168, 2013.

8 Ahn JY, Jung EY, Kwun HJ, Lee CW, Sung YC and Jang KL: Dual effects of hepatitis B virus $X$ protein on the regulation of cell-cycle control depending on the status of cellular p53. J Gen Virol 83: 2765-2772, 2002.
9 Mizushima N, Levine B, Cuervo AM and Klionsky DJ: Autophagy fights disease through cellular self-digestion. Nature 451: 1069-1075, 2008.

10 Gurkar AU, Chu K, Raj L, Bouley R, Lee SH, Kim YB, Dunn SE, Mandinova A and Lee SW: Identification of ROCK1 kinase as a critical regulator of Beclin1-mediated autophagy during metabolic stress. Nat Comm 4: 2189, 2013.

$11 \mathrm{Liu} \mathrm{J}$ and Debnath J: The evolving, multifaceted roles of autophagy in cancer. Adv Cancer Res 130: 1-53, 2016.

12 Sir D, Tian Y, Chen WL, Ann DK, Yen TS and Ou JH: The early autophagic pathway is activated by hepatitis B virus and required for viral DNA replication. Proc Natl Acad Sci USA 107: 4383-4388, 2010.

13 Kinghorn AD, Chin YW and Swanson SM: Discovery of natural product anticancer agents from biodiverse organisms. Curr Opin Drug Discov Devel 12: 189-196, 2009.

14 Ovesna Z, Vachalkova A, Horvathova K and Tothova D: Pentacyclic triterpenoic acids: New chemoprotective compounds. Minireview. Neoplasma 51: 327-333, 2004.

15 Shih WL, Kuo ML, Chuang SE, Cheng AL and Doong SL: Hepatitis B virus X protein inhibits transforming growth factorbeta -induced apoptosis through the activation of phosphatidylinositol 3-kinase pathway. J Biol Chem 275: 25858-25864, 2000.

16 Doong SL, Lin MH, Tsai MM, Li TR, Chuang SE and Cheng AL: Transactivation of the human MDR1 gene by hepatitis B virus X gene product. J Hepatol 29: 872-878, 1998.

17 Wu HY, Chang CI, Lin BW, Yu FL, Lin PY, Hsu JL, Yen CH, Liao MH and Shih WL: Suppression of hepatitis B virus $\mathrm{X}$ protein-mediated tumorigenic effects by ursolic Acid. J Agric Food Chem 59: 1713-1722, 2011.

18 Lin PY, Chang CD, Chen YC and Shih WL: RhoA/ROCK1 regulates Avian Reovirus S1133-induced switch from autophagy to apoptosis. BMC Vet Res 11: 103, 2015.

19 Tang H, Da L, Mao Y, Li Y, Li D, Xu Z, Li F, Wang Y, Tiollais $\mathrm{P}, \mathrm{Li} \mathrm{T}$ and Zhao M: Hepatitis B virus X protein sensitizes cells to starvation-induced autophagy via up-regulation of beclin 1 expression. Hepatology 49: 60-71, 2009.

20 Heinemeyer T, Wingender E, Reuter I, Hermjakob H, Kel AE, Kel OV, Ignatieva EV, Ananko EA, Podkolodnaya OA, Kolpakov FA, Podkolodny NL and Kolchanov NA: Databases on transcriptional regulation: TRANSFAC, TRRD and COMPEL. Nucleic Acids Res 26: 362-367, 1998.

21 Shih WL, Liao MH, Yu FL, Lin PY, Hsu HY and Chiu SJ: AMF/PGI transactivates the MMP-3 gene through the activation of Src-RhoA-phosphatidylinositol 3-kinase signaling to induce hepatoma cell migration. Cancer Lett 270: 202-217, 2008.

22 Aaltonen V, Koivunen J, Laato M and Peltonen J: PKC inhibitor Go6976 induces mitosis and enhances doxorubicin-paclitaxel cytotoxicity in urinary bladder carcinoma cells. Cancer Lett 253: 97-107, 2007.

23 Liu HJ, Lin PY, Wang LR, Hsu HY, Liao MH and Shih WL: Activation of small GTPases RhoA and Rac1 is required for avian reovirus p10-induced syncytium formation. Mol Cells 26: 396-403, 2008.

24 Becker SA, Lee TH, Butel JS and Slagle BL: Hepatitis B virus $\mathrm{X}$ protein interferes with cellular DNA repair. J Virol 72: 266$272,1998$.

25 Liu J: Pharmacology of oleanolic acid and ursolic acid. J Ethnopharmacol 49: 57-68, 1995. 
26 Kapoor NR, Ahuja R, Shukla SK and Kumar V: The HBx protein of hepatitis $\mathrm{B}$ virus confers resistance against nucleolar stress and anti-cancer drug-induced p53 expression. FEBS Lett 587: 1287-1292, 2013

27 Liu Y, Lou G, Wu W, Zheng M, Shi Y, Zhao D and Chen Z: Involvement of the NF-kappaB pathway in multidrug resistance induced by HBx in a hepatoma cell line. J Viral Hepat 18: e439446, 2011.

28 Takahashi Y, Coppola D, Matsushita N, Cualing HD, Sun M, Sato Y, Liang C, Jung JU, Cheng JQ, Mule JJ, Pledger WJ and Wang HG: Bif-1 interacts with Beclin 1 through UVRAG and regulates autophagy and tumorigenesis. Nat Cell Biol 9: 11421151, 2007.

29 Liu YQ, Ji Y, Li XZ, Tian KL, Young CY, Lou HX and Yuan HQ: Retigeric acid B-induced mitophagy by oxidative stress attenuates cell death against prostate cancer cells in vitro. Acta Pharmacol Sin 34: 1183-1191, 2013.

30 Guo JY, Chen HY, Mathew R, Fan J, Strohecker AM, KarsliUzunbas G, Kamphorst JJ, Chen G, Lemons JM, Karantza V, Coller HA, Dipaola RS, Gelinas C, Rabinowitz JD and White E: Activated Ras requires autophagy to maintain oxidative metabolism and tumorigenesis. Gene Dev 25: 460-470, 2011.

31 Lozy F and Karantza V: Autophagy and cancer cell metabolism. Semin Cell Dev Biol 23: 395-401, 2012.

32 Raza A and Sood GK: Hepatocellular carcinoma review: Current treatment, and evidence-based medicine. World J Gastroenterol 20: 4115-4127, 2014.

33 Rawat S and Bouchard MJ: The hepatitis B virus (HBV) HBx protein activates AKT to simultaneously regulate $\mathrm{HBV}$ replication and hepatocyte survival. J Virol 89: 999-1012, 2015.

34 Kabeya Y, Mizushima N, Ueno T, Yamamoto A, Kirisako T, Noda T, Kominami E, Ohsumi Y and Yoshimori T: LC3, a mammalian homologue of yeast Apg8p, is localized in autophagosome membranes after processing. EMBO J 19: 5720-5728, 2000.
35 Marino G, Niso-Santano M, Baehrecke EH and Kroemer G: Self-consumption: The interplay of autophagy and apoptosis. Nature Rev Mol Cell Biol 15: 81-94, 2014.

36 Kang R, Zeh HJ, Lotze MT and Tang D: The Beclin 1 network regulates autophagy and apoptosis. Cell death and differentiation 18: 571-580, 2011

37 Salminen A, Kaarniranta K and Kauppinen A: Beclin 1 interactome controls the crosstalk between apoptosis, autophagy and inflammasome activation: Impact on the aging process. Ageing Res Rev 12: 520-534, 2013.

38 Webber JL and Tooze SA: Coordinated regulation of autophagy by p38alpha MAPK through mAtg9 and p38IP. EMBO J 29: 2740, 2010.

$39 \mathrm{Ke}$ PY and Chen SS: Activation of the unfolded protein response and autophagy after hepatitis $\mathrm{C}$ virus infection suppresses innate antiviral immunity in vitro. J Clin Invest 121: 37-56, 2011.

40 Shrivastava S, Raychoudhuri A, Steele R, Ray R and Ray RB: Knockdown of autophagy enhances the innate immune response in hepatitis C virus-infected hepatocytes. Hepatology 53: 406414, 2011.
Received August 24, 2016

Revised September 6, 2016

Accepted September 7, 2016 